\title{
Functions of PUF Family RNA-Binding Proteins in Aspergillus nidulans
}

\author{
Sung-Hun Son ${ }^{1}$, Seo-Yeong Jang ${ }^{2}$, and Hee-Soo Park ${ }^{1,2 *}$ \\ ${ }^{1}$ School of Food Science and Biotechnology, Kyungpook National University, Daegu 41566, Republic of Korea \\ ${ }^{2}$ Department of Integrative Biology, Kyungpook National University; Daegu 41566, Republic of Korea
}

RNA-binding proteins are involved in RNA metabolism and posttranscriptional regulation of various fundamental biological processes. The PUF family of RNA-binding proteins is highly conserved in eukaryotes, and its members regulate gene expression, mitochondrial biogenesis, and RNA processing. However, their biological functions in Aspergillus species remain mostly unknown in filamentous fungi. Here we have characterized the puf genes in the model organism Aspergillus nidulans. We generated deletion mutant strains for the five putative puf genes present in the A. nidulans genome and investigated their developmental phenotypes. Deletion of pufA or pufE affected fungal growth and asexual development. pufA mutants exhibited decreased production of asexual spores and reduced mRNA expression of genes regulating asexual development. The pufE deletion reduced colony growth, increased formation of asexual spores, and delayed production of sexual fruiting bodies. In addition, the absence of pufE reduced both sterigmatocystin production and the mRNA levels of genes in the sterigmatocystin cluster. Finally, pufE deletion mutants showed reduced trehalose production and lower resistance to thermal stress. Overall, these results demonstrate that PufA and PufE play roles in the development and sterigmatocystin metabolism in A. nidulans.

Keywords: PUF domain, RNA-binding protein, asexual development, sterigmatocystin, conidia

Received: January 8, 2021 Accepted: March 10, 2021

First published online: March 12, 2021

*Corresponding author Phone: +82-53-950-5751 Fax: +82-53-950-6750 E-mail:phsoo97@knu.ac.kr

Supplementary data for this paper are available on-line only at http://jmb.or.kr.

pISSN 1017-7825 eISSN 1738-8872

Copyright(C) 2021 by The Korean Society for Microbiology and Biotechnology

\section{Introduction}

Aspergillus nidulans is a model filamentous fungus widely used in fungal development and metabolism studies $[1,2]$. A. nidulans propagates through both asexual and sexual spores, but asexual spores are their primary reproductive particle [3,4]. Spores germinate to form germ tubes and a web-like mass of fungal hyphae [5]. These hyphae acquire developmental competence and undergo asexual or sexual development, depending on the environmental conditions [6,7]. For example, light can induce the formation of asexual structures-the conidiophores-but sexual fruiting bodies-the cleistothecia-are formed in dark conditions $[8,9]$. These reproductive processes are regulated by a myriad of transcription factors and several signaling pathways [10, 11]. In asexual development, $\mathrm{BrlA}, \mathrm{AbaA}$, and Wet $\mathrm{A}$ are central transcription factors that control mRNA expression of development-related genes $[6,12]$. BrlA is a $\mathrm{C}_{2} \mathrm{H}_{2}$ zinc-finger transcription factor that controls mRNA levels of $a b a \mathrm{~A}$ and other developmental genes [13]. AbaA induces mRNA expression of three key transcription factors: WetA, VosA, and VelB [14-16]. These transcription factors regulate asexual spore maturation, trehalose biosynthesis, and conidial stress tolerance $[15,17]$. Fungal species produce various primary and secondary metabolites during development [18]. Secondary metabolites that have detrimental effects on humans are called mycotoxins $[19,20]$. Sterigmatocystin produced by A. nidulans is a precursor aflatoxin and is considered as a group 2B carcinogen [21]. Sterigmatocystin biosynthesis depends on a gene cluster of which the AflR transcription factor is a key regulator [22-24]. Other important genes involved in sterigmatocystin production include MtfA, VeA, LaeA, and McrA [25-28].

RNA-binding proteins play essential roles in the posttranscriptional regulation of gene expression in eukaryotes [29-31]. In fungi, RNA-binding proteins participate in various fundamental processes, including development, stress responses, filamentation, and pathogenesis [32, 33]. The PUF (Pumilio/Fem-3 binding factor) family of canonical RNA-binding proteins is conserved among most eukaryotic systems [34]. The Pumilio in Drosophila melanogaster and Fem-3 binding factor (FBF) in Caenorhabditis elegans are the founding members of the PUF family proteins [35]. They contain several repeated PUF domains, which interact with sequencespecific RNA elements of their target. [35]. In Ascomycota fungi, PUF proteins recognize 8- to 10-nucleotide binding elements, reducing translational efficiency or by increasing its decay [36,37]. Most of the research on the RNA targets and the biological roles of the PUF proteins have been carried out in Saccharomyces cerevisiae [35, 38, 39]. S. cerevisiae contains six PUF proteins involved in posttranscriptional processes, mating type switching, lifespan, thermotolerance, cell wall integrity, and mitochondrial biogenesis [35, 40]. For example, PUF6 targets 
mRNA of Ash1, which is involved in budding [41]. However, little is known about PUF proteins in filamentous fungi, particularly in Aspergillus species.

RNA-binding proteins, including SwoK, FabM, PbpA, and RrmA, have been characterized in A. nidulans [4246]. These RNA-binding proteins regulate fungal development and primary and secondary metabolisms. In this study, we characterized the $A$. nidulans PUF family of RNA-binding proteins. The A. nidulans genome encodes five putative proteins containing PUF repeated motifs (PufA to E). With phenotypic and genomic analyses, we show that PufE is required for normal fungal development, spore maturation, and sterigmatocystin production in A. nidulans.

\section{Materials and Methods}

\section{Strains, Media, and Growth Conditions}

The A. nidulans strains used in this study are listed in Table 1. Fungal strains were grown on solid or liquid minimal media with $1 \%$ glucose (MMG) [47]. For auxotroph mutants, uridine (Acros Organics, USA), uracil (Acros Organics), and pyridoxine (Sigma-Aldrich, USA) were supplemented in the media. Sexual media (SM) was used to induce sexual development [48]. To observe developmental phenotypes, the strains were point inoculated in solid MMG or SM and cultured at $37^{\circ} \mathrm{C}$ for 5-7 days. For sterigmatocystin extraction, the strains were cultured in liquid complete media $(\mathrm{CM})$ at $30^{\circ} \mathrm{C}$ for 7 days. To collect fresh conidia, each strain was incubated in solid MMG at $37^{\circ} \mathrm{C}$ for 2 days. For amplification of the plasmid used to generate the complemented strains, Escherichia coli DH5a (Enzynomics, Korea) cells were grown in a Luria-Bertani medium (BD Difco, USA) with ampicillin $(100 \mu \mathrm{g} / \mathrm{ml})$ (Sigma-Aldrich).

\section{Construction of pufDeletion Mutant Strains}

The oligonucleotides used in this study are listed in Table 2. To generate deletion mutants for puf genes, disruption cassettes of each gene were prepared using the double-joint PCR method [49]. First, the 5' and 3' regions of each pufgene were amplified from the A. nidulans FGSC A4 genomic DNA template, using DF/TailR and DR/TailF primer pairs. The A. fumigatus pyrG (AfupyrG) was used as a selection marker and was amplified from the A. fumigatus genomic DNA template using a 5'AfupyrG_F (OHS089)/3' AfupyrG_R (OHS090) primer pair. The amplified 5' and 3' regions of the pufgenes and the AfupyrG selection markers were linked through PCR and amplified using an NF/NR primer pair to obtain the puf gene disruption cassettes. The deletion cassette was introduced into protoplasts of the A. nidulans RJMP1.59 strain, made with the Vinoflow FCE lysing enzyme (Novozymes, Bagsvaerd, Denmark) [47]. After culturing transformed cells in the selection media (MMG without uridine and uracil), transformants were confirmed by PCR followed by restriction enzyme digestion.

\section{Generation of the Complemented pufA and pufE Strains}

To generate complemented strains, the regions containing the promoter and open reading frame sequences of pufA or pufE were amplified with the OHS1136/OHS1137 or OHS1380/OHS1381 primer pairs, respectively, and digested with NotI. These DNA fragments were inserted into pHS13 [14], generating pSH4.1 or pSH5.1, for pufA or pufE, respectively. The pSH4.1 or pSH5.1 plasmid was introduced into the $\triangle p u f A$ (TSH8.1) or $\triangle p u f E$ (TSH27.1) strains to give rise to TSH17 and TSH28, respectively.

\section{Quantitative Reverse-Transcription PCR (qRT-PCR) Analysis}

For qRT-PCR analysis, the samples were prepared as described previously [50]. Asexual spores of each strain were inoculated in liquid $M M G$ and cultured at $37^{\circ} \mathrm{C}$ for $16 \mathrm{~h}$. Cultured mycelia were squeeze-dried and stored at $-80^{\circ} \mathrm{C}$ until RNA extraction. For the asexual developmental samples, the mycelia were filtered using Miracloth (Calbiochem, USA) and monolayered onto solid MMG. The plates were incubated for 12 and $24 \mathrm{~h}$ in light conditions to induce asexual development. After squeeze-drying, the samples were stored at $-80^{\circ} \mathrm{C}$ until RNA extraction. For conidia, each strain was cultured in solid $M M G$ at $37^{\circ} \mathrm{C}$ for 2 days and then the conidia were collected using Miracloth and stored at $-80^{\circ} \mathrm{C}$ until RNA extraction.

Total RNA was extracted as described previously [50]. The samples were homogenized in a TRIzol reagent

Table 1. Aspergillus strains used in this study.

\begin{tabular}{|c|c|c|}
\hline Strain name & Relevant genotype & References \\
\hline FGSC A4 & A. nidulans wild type, $v e A^{+}$ & FGSC $^{a}$ \\
\hline RJMP1.59 & pyrG89; pyroA4; veA ${ }^{+}$ & {$[55]$} \\
\hline TNJ36 & pyrG89; AfupyrG ${ }^{+} ;$pyroA4; veA $^{+}$ & {$[56]$} \\
\hline TSH8.1 3 & pyrG89; pyroA4; $\triangle$ pufA::AfupyrG $G^{+} ;$veA $^{+}$ & This study \\
\hline TSH10.1 3 & pyrG89; pyroA4; $\triangle$ pufB::AfupyrG ${ }^{+} ;$veA $^{+}$ & This study \\
\hline TSH11.1 3 & pyrG89; pyroA4; $\Delta$ pufC::AfupyrG $G^{+} ;$veA $^{+}$ & This study \\
\hline TSH9.1 3 & pyrG89; pyroA4; $\Delta$ pufD::AfupyrG ${ }^{+} ;$ve $A^{+}$ & This study \\
\hline TSH27.1 3 & pyrG89; pyroA4; $\triangle$ pufE::AfupyrG ${ }^{+} ;$veA $^{+}$ & This study \\
\hline TSH17.1 3 & pyrG89;pyroA::pufA(p)::pufA::FLAG3x::pyroA ${ }^{b} ; \Delta$ pufA::AfupyrG ${ }^{+} ;$veA $^{+}$ & This study \\
\hline TSH28.1 3 & pyrG89;pyroA::pufE(p)::pufE::FLAG3x::pyroA $A^{b} ; \Delta$ pufE::AfupyrG ${ }^{+} ; v A^{+}$ & This study \\
\hline
\end{tabular}

${ }^{a}$ Fungal Genetic Stock Center

${ }^{\mathrm{b}}$ The $3 / 4$ pyroA marker causes targeted integration at the pyroA locus. 
Table 2. Oligonucleotides used in this study.

\begin{tabular}{|c|c|c|}
\hline Name & Sequence $\left(5^{\prime} \rightarrow 3^{\prime}\right)^{\mathrm{a}}$ & Purpose \\
\hline OHS0089 & GCTGAAGTCATGATACAGGCCAAA & AfupyrG Marker_F \\
\hline OHS0090 & ATCGTCGGGAGGTATTGTCGTCAC & AfupyrG Marker_R \\
\hline OHS0842 & GGA AGCTGACTACGCGGAA & pufA_5'DF \\
\hline OHS0843 & TTCTCCAGCTTTGGCCCT & pufA_3'DR \\
\hline OHS0844 & GGCTTTGGCCTGTATCATGACTTCA ACGGTCAAAAGCTCACCCC & pufA_Rev with $A f-p y r G$ tail R \\
\hline OHS0845 & TTTGGTGACGACAATACCTCCCGAC GTCGGA ATTCTCCCATCGC & $p u f A$ For with $A f-p y r G$ tail F \\
\hline OHS0846 & AAACGAAGGACGGACCAACC & pufA_nested 5'NF \\
\hline OHS0847 & GTATCCCGACGCTTCGATGA & pufA_nested 3' NR \\
\hline OHS0848 & CTGGAGCTATCACACCGTCT & pufA_RT_F \\
\hline OHS0849 & CGATTGACAGTCGCATGGTT & pufA_RT_R \\
\hline OHS0834 & ATCGCGTCCTCGTCCTATC & pufB_5'DF \\
\hline OHS0835 & ATCCTCTAACCGTTCGCGC & pufB_3'DR \\
\hline OHS0836 & GGCTTTGGCCTGTATCATGACTTCA TTATTGGAGGGACCGACGAC & pufB_Rev with $A f-p y r G$ tail R \\
\hline OHS0837 & TTTGGTGACGACAATACCTCCCGAC GCCTACATCGAGGGTCGTT & $p u f B \_$For with $A f-p y r G$ tail $\mathrm{F}$ \\
\hline OHS0838 & ACACAATTCTTGGCTCCCCG & pufB_nested 5' NF \\
\hline OHS0839 & CATAGCCTGATGTGCCGCT & pufB_nested 3' NR \\
\hline OHS0840 & CTCCTGTCCAGAATCGCTCT & pufB_RT_F \\
\hline OHS0960 & ATCCTGATTGTGCCGGGTAA & pufB_RT_R \\
\hline OHS0850 & AGCCGCAACCTCCTACAA & pufC_5'DF \\
\hline OHS0851 & CCGCTAGATGTTGCGACCT & pufC_3' DR \\
\hline OHS0852 & GGCTTTGGCCTGTATCATGACTTCA CGATAGGGCACAATGGGCT & pufC_Rev with $A f-p y r G$ tail R \\
\hline OHS0853 & TTTGGTGACGACAATACCTCCCGAC CCGGTA AGACTATTC AGC & pufC_For with $A f-p y r G$ tail F \\
\hline OHS0854 & GCCTAACGAAGGGTTGGAGT & pufC_nested 5' NF \\
\hline OHS0855 & TAAAGACTTGGACCGGGGCT & pufC_nested 3' NR \\
\hline OHS0856 & CCAAAGAAGTTGCGCGTTTC & pufC_RT_F \\
\hline OHS0857 & CGGGTTTGTGCTAGTTCCTG & pufC_RT_R \\
\hline OHS0826 & ATGTTGCGCGGCCTATTG & pufD_5'DF \\
\hline OHS0827 & AGCATGCGGTACTTCGAGT & pufD_3'DR \\
\hline OHS0828 & GGCTTTGGCCTGTATCATGACTTCA CTG ATGAGAAGAGCGGTGACG & pufD_Rev with $A f-p y r G$ tail R \\
\hline OHS0829 & TTTGGTGACGACAATACCTCCCGAC CGAACAACAATCTTGGCGCTC & pufD_For with $A f-p y r G$ tail F \\
\hline OHS0830 & GCGAAAGCAGGCATATCTGG & pufD_nested 5'NF \\
\hline OHS0831 & CACCTCGATCGGCAGCATA & pufD_nested 3'NR \\
\hline OHS0832 & ACAACGTCAGCTTGGGTAGA & pufD_RT_F \\
\hline OHS0833 & TGCAATGTCGTAGGCTGAGA & pufD_RT_R \\
\hline OHS1330 & CAACCCCATTGAGCCTCAG & pufE_5'DF \\
\hline OHS1331 & GGGATTAGCACAGCACGTG & pufE_3'DR \\
\hline OHS1332 & GGCTTTGGCCTGTATCATGACTTCA GTGGCTCCA AGGTCTGTCT & pufE_Rev with $A f-p y r G$ tail R \\
\hline OHS1333 & TTTGGTGACGACAATACCTCCCGAC AGGGCCATCATTTCGCCA & pufE_For with $A f$-pyrG tail F \\
\hline OHS1334 & CTCGATTTCCCGTGAGTCTTTCG & pufE_nested 5' NF \\
\hline OHS1335 & TCA ATA AGCTCGGACTGCCTG & pufE_nested 3' NR \\
\hline OHS1336 & GAACCTGCTTAGGGAGTGGT & pufE_RT_F \\
\hline OHS1337 & TTCGAGGAGGAAGTGCATGA & pufE_RT_R \\
\hline OHS1136 & AATTGCGGCCGCACGGTACACACTTTACCCGG & C'pufA_Not1F \\
\hline OHS1137 & AATTGCGGCCGCGAAGGTATAGGCAAAGTTCAGCGC & C'pufA_Not1R \\
\hline OHS1380 & AATTGCGGCCGCGAAATCGCCATATGCGCC & C'pufE_Not1F \\
\hline OHS1381 & AATTGCGGCCGCCGCAATCTTCTCCAACAATAACTT & C'pufE_Not1R \\
\hline OHS0580 & CAAGGCATGCATCAGTACCC & brlA_RT_F \\
\hline OHS0581 & AGACATCGAACTCGGGACTC & brlA_RT_R \\
\hline OHS0779 & ATTGACTGGGAAGCGAAGGA & $a b a A \_$RT_F \\
\hline OHS0780 & CTGGGCAGTTGAACGATCTG & $a b a A \_R T \_R$ \\
\hline OHS0599 & GCGCGAAGAAGACTTCAAC & aflR_RT_F \\
\hline OHS0600 & TGCAATAACTGCCGACGAC & aflR_RT_R \\
\hline OHS0946 & GGATCTGCCAAAGCGAACAT & stcA_RT_F \\
\hline OHS0947 & CCACAGTGAGGAGGAATGGT & stcA_RT_R \\
\hline OHS0604 & GCTACTGTTCCAGGCGACTA & $s t c E \_R T \_F$ \\
\hline OHS0605 & CACAGCTCTCCATCTCGGTA & $s t c E \_R T \_\mathrm{R}$ \\
\hline OHS0602 & CGCATCATCCTCACAAGTTC & stcU_RT_F \\
\hline OHS0603 & TGACCGTGATCTTCTTGTCG & stcU_RT_R \\
\hline
\end{tabular}

Tail sequences are shown in italics. Restriction enzyme sites are in bold.

(Invitrogen, USA) and $0.5 \mathrm{~mm}$ zirconia/silica beads (RPI, USA), using a Mini-Bead Beater (BioSpec Product, USA). Then, the supernatant was separated by centrifugation, transferred into a new tube, and mixed with cold isopropanol. The pellets were resuspended with RNase-free water (Promega, USA) and treated with DNase I 
(Promega) to remove DNA. cDNA was synthesized with the GoScript Reverse Transcriptase (Promega). Quantitative PCR was conducted using iTaq Universal SYBR Green Supermix (Bio-Rad, USA) and CFX96 Touch Real-Time PCR (Bio-Rad). The expression levels for each target gene were calculated using the $2^{-\Delta \Delta C T}$ method. The $\beta$-actin gene expression was used as an endogenous control.

\section{Quantification of Asexual spores and Cleistothecia}

To test conidia production, the strains were inoculated in solid MMG at $37^{\circ} \mathrm{C}$ for 5 days, in light or dark conditions. Colony morphology was photographed with a Pentax MX-1 digital camera and a Zeiss Lab.A1 microscope equipped with an AxioCam 105c camera and AxioVision (Rel. 4.9) digital imaging software. For quantification, conidia were collected from the plate using $\mathrm{ddH}_{2} \mathrm{O}$ and Miracloth. The number of conidia was determined in triplicate for each strain with a hemocytometer.

For the formation of cleistothecia, each strain was point inoculated in solid SM and cultured in dark conditions at $37^{\circ} \mathrm{C}$ for 7 or 14 days. Cultured plates were washed with $100 \%$ ethanol, and the size of cleistothecia was measured using a Zeiss Lab.A1 microscope equipped with an AxioCam 105c camera and AxioVision (Rel. 4.9) digital imaging software. To measure the number of ascospores, cleistothecia were collected from each strain and broken into e-tubes. Then, they were diluted with $\mathrm{ddH}_{2} \mathrm{O}$ and measured with a hemocytometer. The number of ascospores was counted in triplicate for each strain.

Sterigmatocystin (ST) Extraction and Thin Layer Chromatography (TLC) Analysis. To extract ST from each strain, approximately $10^{5}$ conidia were inoculated into $5 \mathrm{ml} \mathrm{liquid} \mathrm{CM}$ and cultured at $30^{\circ} \mathrm{C}$ for 7 days in dark conditions [51]. Then, $5 \mathrm{ml}$ of chloroform was added to the cultured samples and the samples were mixed. The organic phases were separated through centrifugation, transferred to a new glass vial, and evaporated overnight. After complete evaporation, $200 \mu \mathrm{l}$ of chloroform was added, and approximately $6 \mu \mathrm{l}$ of the sample was loaded onto a TLC silica plate (Kiesel gel 60, $0.25 \mathrm{~mm}$; Merck, Germany). Commercial sterigmatocystin (Sigma-Aldrich) was used as a loading control. To separate the samples, the TLC plate was developed in a chloroform: ethyl acetate $(9: 1 \mathrm{v} / \mathrm{v})$ solution and treated with $1 \%$ aluminum hydroxide hydrate (Sigma-Aldrich). The TLC plate was observed under UV light $(366 \mathrm{~nm})$ and the relative intensity of the ST spots was calculated using ImageJ. The experiments were conducted in a triplicate for each strain.

\section{Trehalose Assay}

The trehalose content in conidia was determined as previously described [52]. Briefly, the strains were incubated in solid MMG at $37^{\circ} \mathrm{C}$ for 2 days and approximately $2 \times 10^{8}$ conidia were collected with $\mathrm{ddH}_{2} \mathrm{O}$ with $0.01 \%$ Triton X-100 (Sigma-Aldrich). After centrifugation, the supernatant was removed and resuspended in $200 \mu \mathrm{l}$ of $\mathrm{ddH}_{2} \mathrm{O}$ with $0.01 \%$ Triton $\mathrm{X}-100$ and heated at $95^{\circ} \mathrm{C}$ for $20 \mathrm{~min}$. The samples were separated by centrifugation, and two equal volumes of supernatant per strain were transferred to a new tube. A volume of $0.2 \mathrm{M}$ sodium citrate ( $\mathrm{pH}$ 5.5) was added to the two prepared samples. Trehalase ( $3 \mathrm{mU}$, Sigma-Aldrich) was added to one of the two samples (the other served as the negative control), and samples were incubated at $37^{\circ} \mathrm{C}$ for $8 \mathrm{~h}$ to decompose trehalose into glucose. The amount of glucose present was measured using a Glucose Assay Kit (Sigma-Aldrich). The glucose assays of were done in triplicate for each strain.

\section{Spore Viability Assay}

The method to test spore viability was described previously [51]. Briefly, strains were incubated in solid MMG at $37^{\circ} \mathrm{C}$ for 2 or 10 days; subsequently, approximately $10^{3}$ conidia were collected with $\mathrm{ddH}_{2} \mathrm{O}$ containing $0.01 \%$ Triton X-100 (Sigma-Aldrich). Approximately 100 conidia were spread onto solid MMG and cultured for 2 days at $37^{\circ} \mathrm{C}$. Spore viability was measured by determining the number of colonies formed.

\section{Thermal Tolerance Assay}

Thermal tolerance tests were performed as previously described [53]. Briefly, approximately $10^{3}$ conidia per ml were incubated at $55^{\circ} \mathrm{C}$ for $15 \mathrm{~min}$ or $30 \mathrm{~min}$. Then, $100 \mu \mathrm{l}$ of the conidia suspension was spread onto solid MMG. After incubation at $37^{\circ} \mathrm{C}$ for 2 days, the number of colony-forming units was counted. Conidia not treated with heat were used as a control. All experiments were carried out in triplicate.

\section{Statistical Analysis}

Student's unpaired $t$-tests were used to evaluate the statistical differences between control and deletion mutants. Data are reported as mean \pm standard deviation. $P$-values $<0.05$ were considered statistically significant.

\section{Results and Discussion \\ pufGenes in A. nidulans}

Comparative genomic analyses found five genes encoding PUF repeat-containing proteins in three Aspergillus species: A. nidulans, A. oryzae, and A. fumigatus [54]. The predicted Aspergillus PUF protein sequences were aligned with the S. cerevisiae Puf1-Puf6 proteins using Clustal Omega (https://www.ebi.ac.uk/Tools/msa/ clustalo/) and MEGA phylogenetic analysis tools. We predicted that AN7474, AN6587, AN4285, AN10071, and AN10173 are the potential homologs of S. cerevisiae Puf1 (identity 46.13\%), Puf3 (identity 41.49\%), Puf3 (identity 26.40\%), Puf4 (identity $47.72 \%$ ), and Puf5 (identity 31.76\%), respectively. Based on these results, A. nidulans puf genes were named pufA (AN7474), pufB (AN6587), pufC (AN4285), pufD (AN10071), and pufE (AN10173) (Fig. 1A). Domain analysis found that all PUF proteins contain three-eight Pumilio-like repeats (SM00025). PufA, similar to ScPuf1 and ScPuf2, contains six Pumilio-like repeats in a row, and PufB, PufC, and PufD have seven or 
A
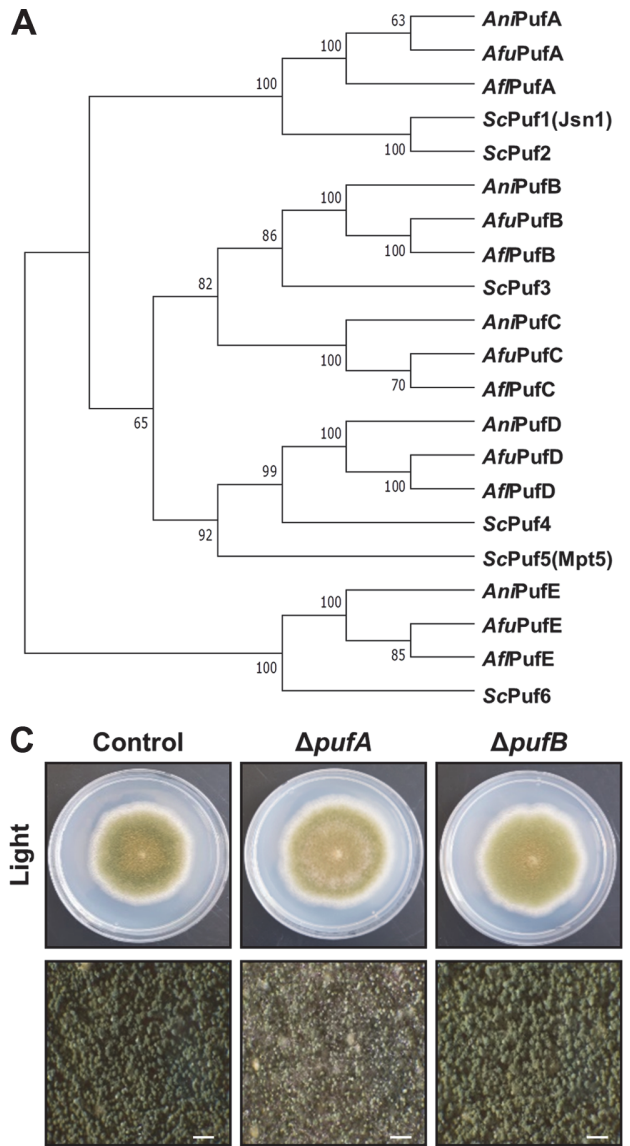

D

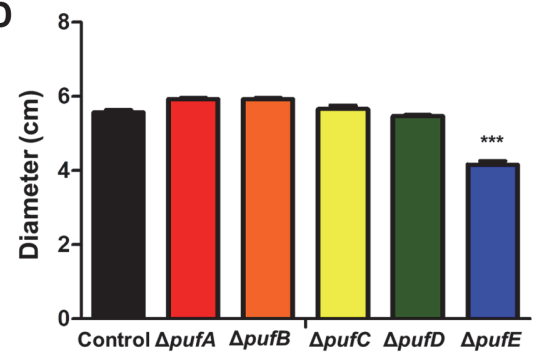

$\Delta p u f B$
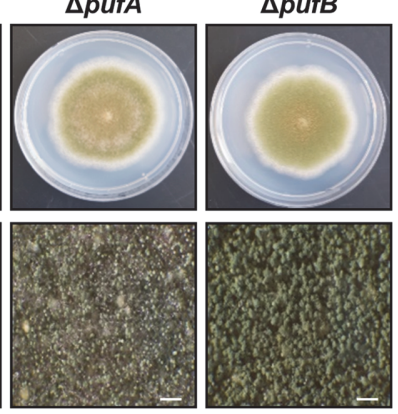

B

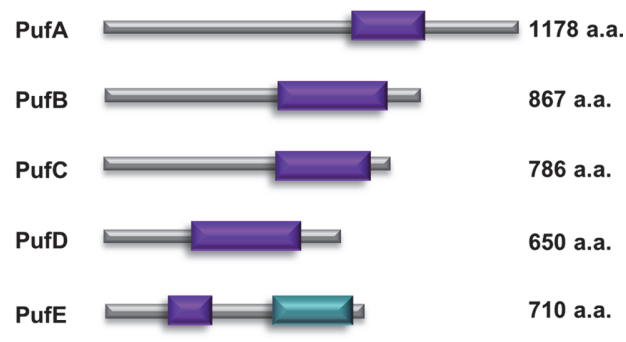

PUF domain $\square$ CPL domain

$\Delta$ pufC

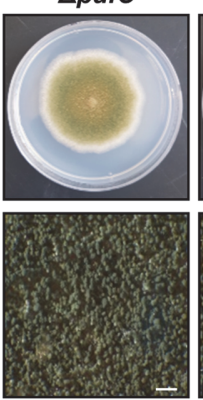

$\Delta p u f D$

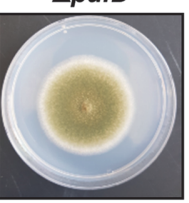

$\Delta$ pufE
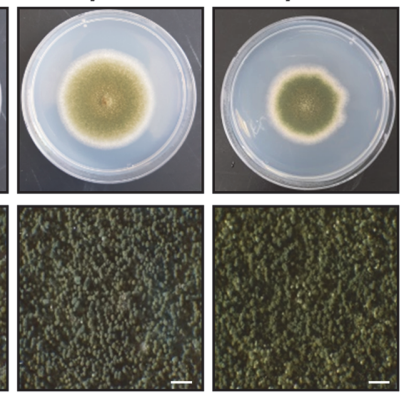

E

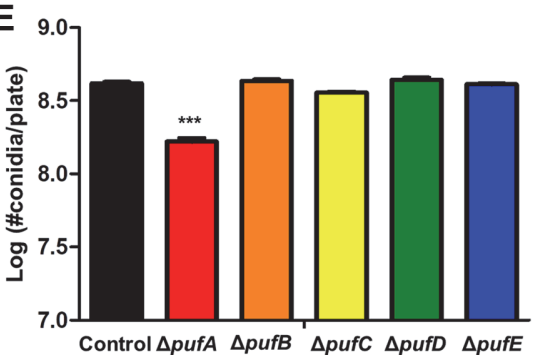

Fig. 1. Summary of the puf genes in A. nidulans. (A) Phylogenetic tree of the putative Puf proteins in A. nidulans FGSC4, A. fumigatus AF293, A. flavus NRRL 3357, and S. cerevisiae SC288. (B) Domain architecture of the putative Puf proteins in A. nidulans. (C) Control (TNJ36) and puf deletion mutant strains grown on MMG agar plates for 5 days at $37^{\circ} \mathrm{C}$ under light condition. The bottom panel shows close-up views of the center of the colony $(\mathrm{bar}=0.25 \mu \mathrm{m})$. (D) Colony diameter of control (TNJ36) and puf deletion mutant strains shown in (A). (E) Number of conidia of control (TNJ36) and puf deletion mutant strains shown in (A).

eight Pumilio-like repeats. Unlike other PUF proteins in A. nidulans, PufE contains three Pumilio-like repeats. And PufE has a CPL domain at the C-terminus region, similar to ScPuf6 (Fig. 1B).

To investigate the roles of the five puf genes in A. nidulans, we generated deletion mutants and examined their colony growth and asexual spore production (Fig. 1C). pufE mutants had reduced colony diameter, and pufA mutants produced fewer asexual spores than control strain (Figs. 1D and 1E). pufB, pufC, and pufD did not show any growth or sporulation phenotypes. We further analyzed the roles of PufA and PufE proteins in A. nidulans.

Deletion of pufA Reduces Asexual Spore Formation. To further examine the role of PufA in asexual development, we generated a pufA complemented strain (C'pufA) and compared its phenotype with that of the mutant (Fig. 2A). Similar to what we reported above (Fig. 1), a deletion of pufA led to decreased production of asexual spores in both light and dark conditions (Fig. 2B). In addition, deletion of pufA affected the mRNA expression of two asexual development regulating genes, brlA and $a b a A$ (Fig. 2C). Transcript levels of brlA and $a b a \mathrm{~A}$ decreased at $24 \mathrm{~h}$ after asexual developmental induction. Asexual spore production and $b r l A$ and $a b a A$ mRNA levels in C' pufA were not significantly different from the control. Overall, these results demonstrate that PufA is required for asexual development in A. nidulans. 


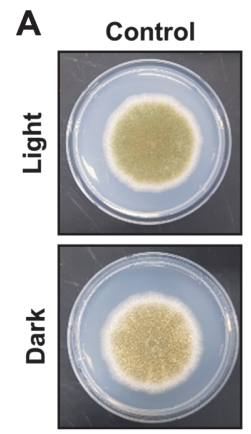

C
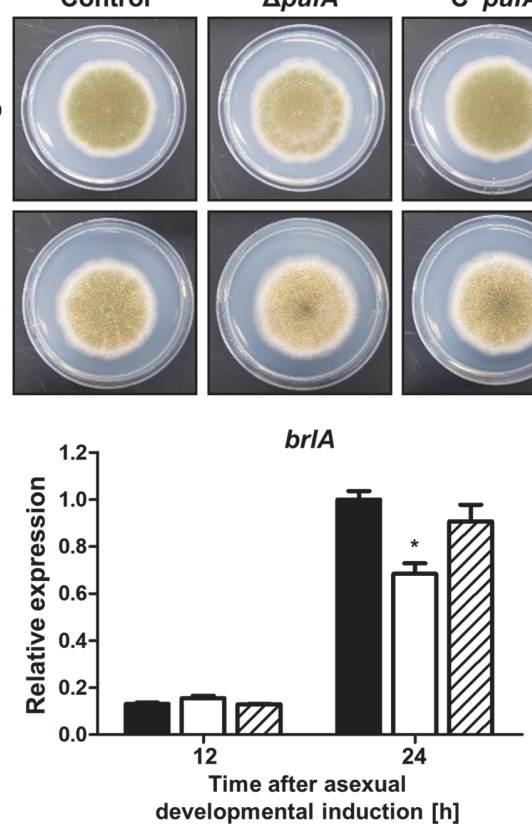

C' pufA

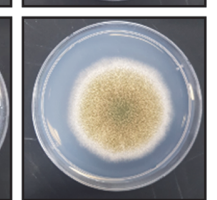

B
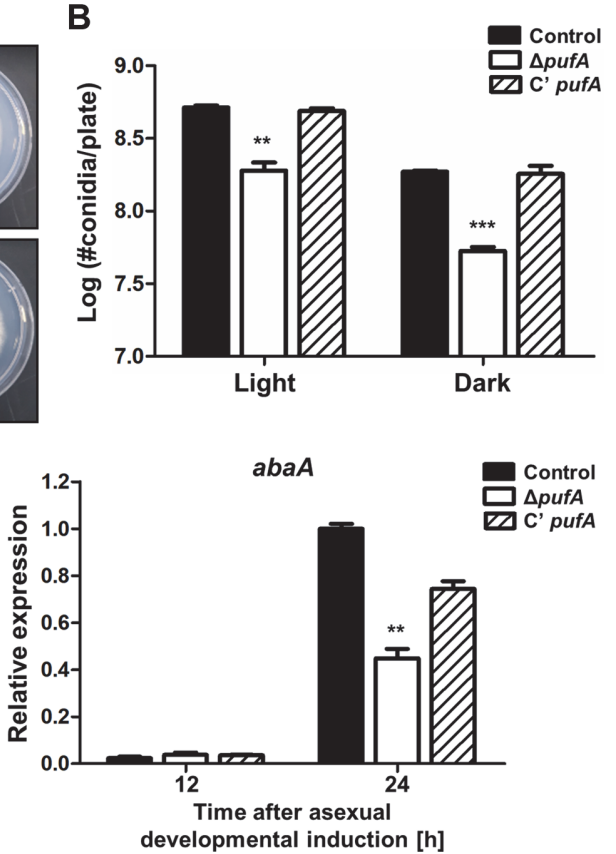

Fig. 2. Phenotypic analysis of the $\Delta p u f A$ mutant strains. (A) Colony photographs of control (TNJ36), $\Delta p u f A$ (TSH8.1), and complemented pufA (C' pufA, TSH17.1) strains point inoculated on solid MMG and grown for 5 days under light or dark conditions. (B) Quantitative analysis of asexual spore production of control (TNJ36), $\Delta p u f A$ (TSH8.1), and C' pufA (TSH17.1) strains shown in (A). (C) mRNA levels of brlA and abaA in control (TNJ36), $\Delta p u f A$ (TSH8.1), and C'pufA (TSH17.1) strains shown in (A). Numbers indicate the time (h) after induction of asexual development. Differences between the control and $\triangle p u f A,{ }^{*} p<0.05,{ }^{* *} p<0.01$, and ${ }^{* *} p<0.001$.

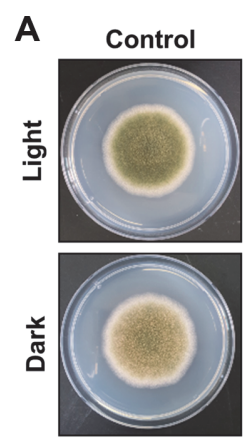

C

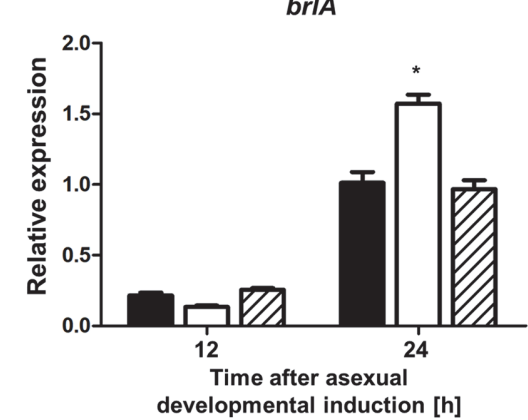

B
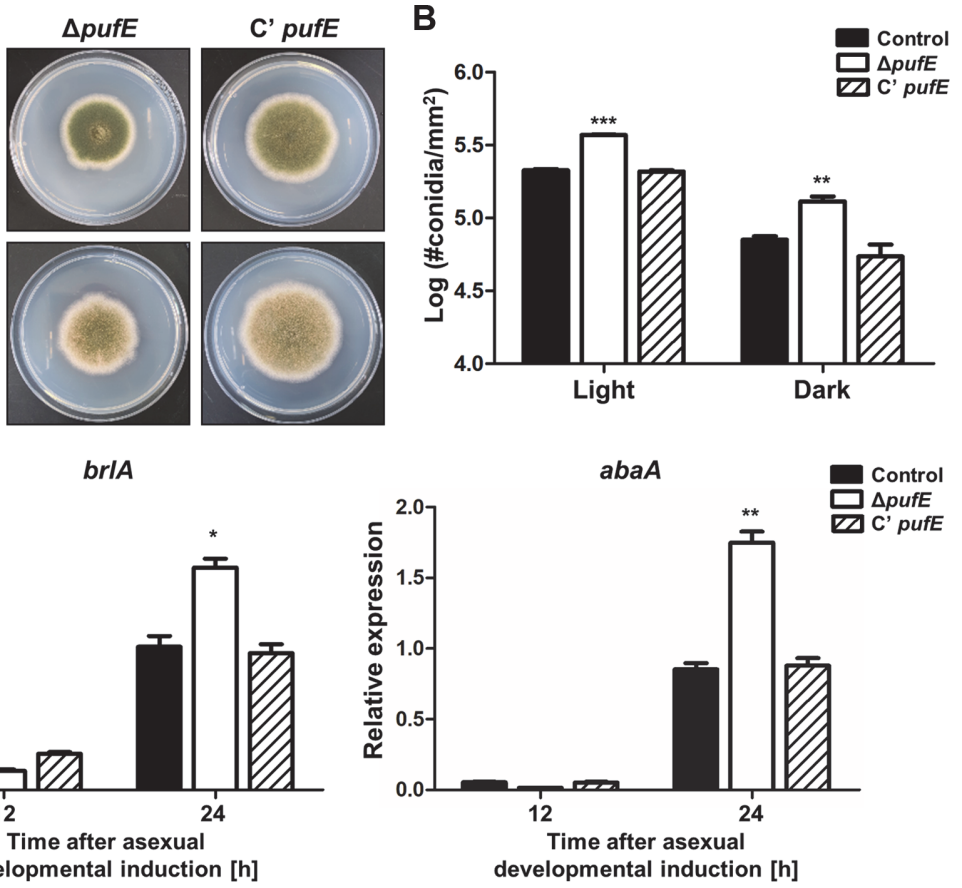

Fig. 3. Asexual developmental analysis of the $\Delta p u f E$ mutant strains. (A) Colony photographs of control (TNJ36), $\triangle p u f E$ (TSH27.1), and the complemented pufE (C'pufE, TSH28.1) strains point inoculated on solid MMG and grown for 5 days under light or dark conditions. (B) Quantitative analysis of asexual spore production of control (TNJ36), $\triangle$ pufE (TSH27.1), and C' pufE (TSH28.1) strains shown in (A). (C) mRNA levels of brlA and abaA in control (TNJ36), $\triangle p u f E$ (TSH27.1), and C' pufE (TSH28.1) strains shown in (A). Numbers indicate the time (h) after induction of asexual development. Differences between the control and $\Delta p u f E,{ }^{*} p<0.05,{ }^{* *} p<0.01$, and ${ }^{* * *} p<0.001$. 
A

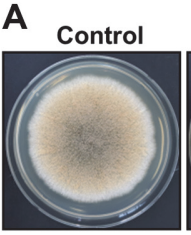

$\triangle$ pufE
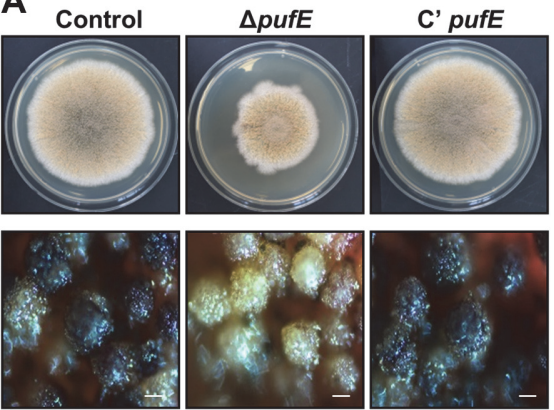

7days
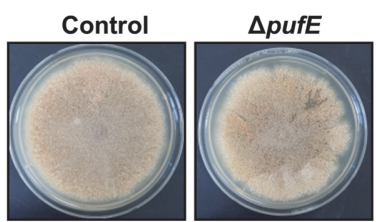

C' pufE
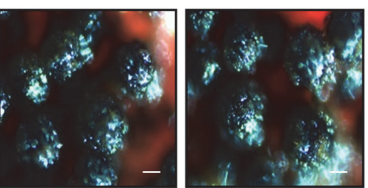

14days
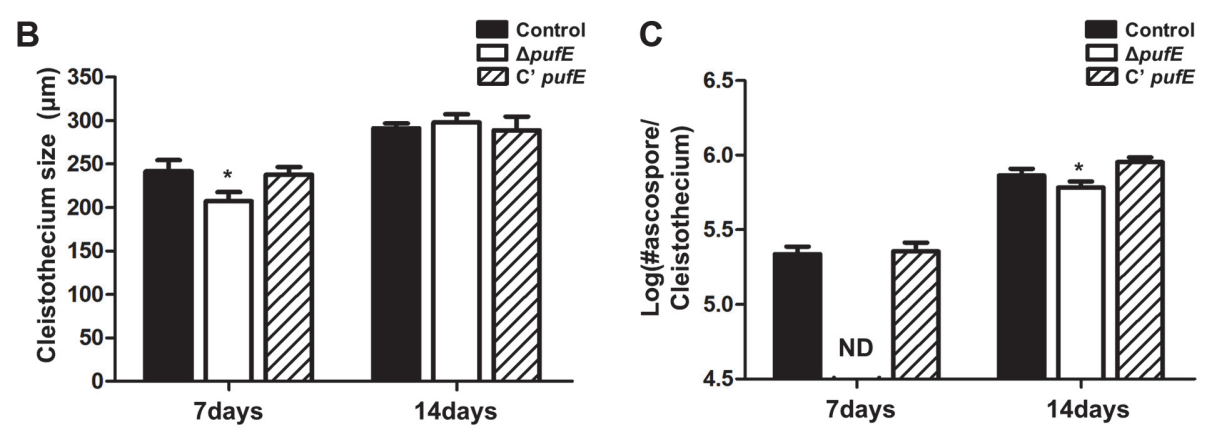

Fig. 4. Sexual development of the $\Delta p u f E$ mutant strains. (A) Sexual fruiting body formation of control (TNJ36), $\Delta p u f E$ (TSH27.1), and C' pufE (TSH28.1) strains point inoculated on solid SM and grown for 7 or 14 days under dark condition. The bottom panel shows close-up views of the center of the plates (Bar $=200 \mu \mathrm{m})$. (B) Size of cleistothecium of control (TNJ36), $\triangle p$ ufE (TSH27.1), and C' pufE (TSH28.1) strains shown in (A). (C) Number of ascospores (log scale) per cleistothecium of control (TNJ36), $\Delta p u f E$ (TSH27.1), and C' $p u f E$ (TSH28.1) strains. Differences between the control and $\Delta p u f E,{ }^{*} p<0.05$. ND, not detected.

PufE is required for normal asexual and sexual development. As shown in Fig. 1, the deletion of pufE resulted in decreased colony diameter but the number of asexual spores in the entire colony appeared similar to the control. To further understand these phenotypes, we calculated the number of asexual spores per area in mutant and complemented strains. $\triangle p u f E$ had decreased colony diameter but produced more asexual spores than the control and 'C' pufE strains in both dark and light conditions (Fig. 3). Additionally, the mRNA levels of asexual development-related genes, $b r l A$ and $a b a A$, increased $24 \mathrm{~h}$ after asexual developmental induction. These results indicate that PufE is essential for fungal development and asexual development in A. nidulans.

We also examined the role of PufE in sexual development in A. nidulans (Fig. 4). Control, $\Delta p u f E$, and C' pufE strains were point inoculated into SM and incubated under the dark condition. At 7 days after incubation, control and C' $p u f E$ strains produced matured sexual fruiting bodies; however, $\Delta p u f E$ sexual fruiting bodies remained

A

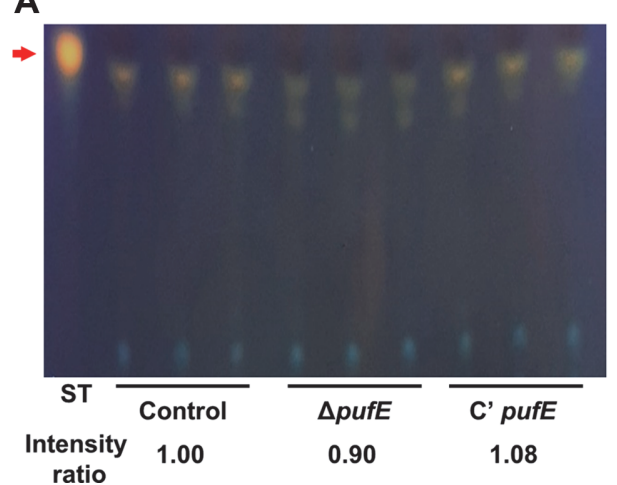

B

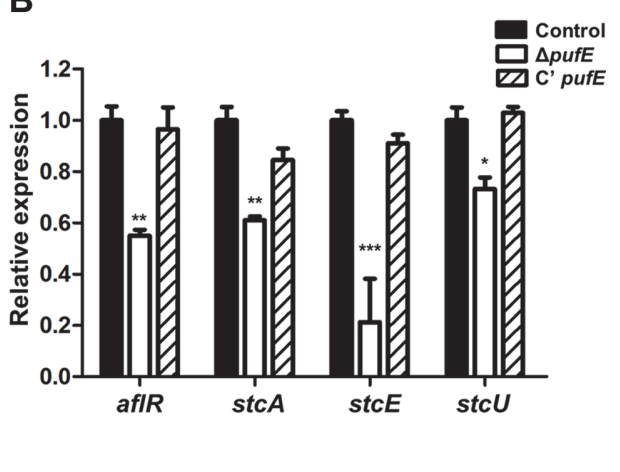

Fig. 5. Sterigmatocystin production in the $\Delta p u f E$ mutant strains. (A) TLC image of sterigmatocystin from control (TNJ36), $\triangle p$ pufE (TSH27.1), and C' pufE (TSH28.1) strains. These strains were cultured into CM for 7 days under dark condition. Arrow indicates sterigmatocystin for standard. (B) mRNA levels of aflR, stcA, stcE, and $s t c U$ in control (TNJ36), $\Delta p u f E$ (TSH27.1), and C'pufE (TSH28.1). Differences between the control and $\Delta$ pufE, ${ }^{*} p<0.05,{ }^{* *} p<0.01$, and ${ }^{* *} p<0.001$. 

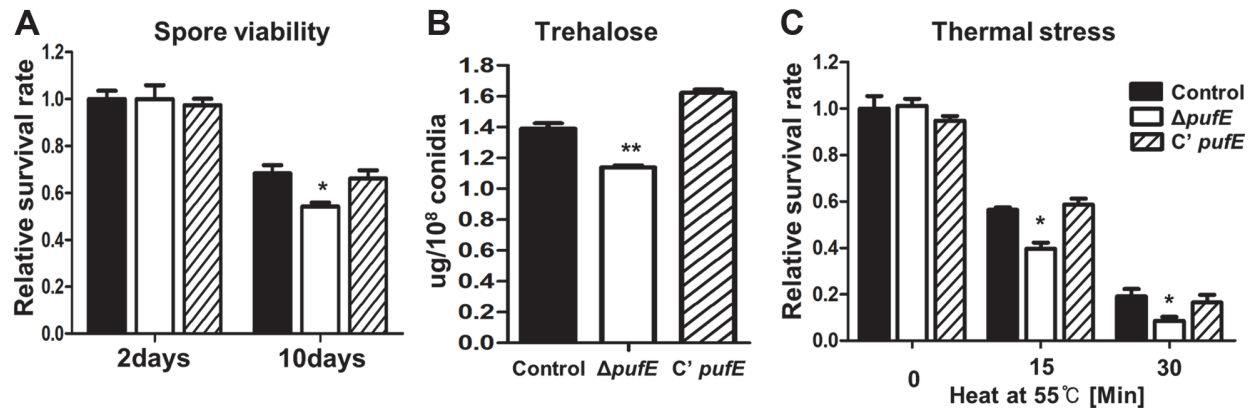

Fig. 6. Spore viability and thermal tolerance of the $\Delta p u f E$ mutant conidia. (A) Spore viability of control (TNJ36), $\triangle$ pufE (TSH27.1), and C' pufE (TSH28.1) strains. (B) Trehalose contents in the 2-day-old conidia (10 ${ }^{8}$ ) of control (TNJ36), $\triangle p u f E$ (TSH27.1), and C' pufE (TSH28.1) strains (measured in triplicate). (C) Heat tolerance of control (TNJ36), $\Delta p u f E$ (TSH27.1), and C'pufE (TSH28.1) conidia (triplicate measurements). Differences between the control and $\Delta$ pufE, ${ }^{*} p<0.05$, ${ }^{* *} p<0.01$, and ${ }^{* * *} p<0.001$.

immature (Fig. 4A). $\triangle p u f E$ cleistothecia were smaller than in control and C' pufE strains (Fig. 4B). Ascospores were not detected in $\triangle p u f E$ cleistothecium but were present in control and C' pufE strains (Fig. 4C). At 14 days, $\triangle p u f E$ strains produced matured cleistothecia; cleistothecium size was similar in all strains. However, $\triangle p u f E$ cleistothecium contained fewer ascospores than in control and C' pufE. These results indicate that PufE is involved in the formation and maturation of sexual fruiting bodies in A. nidulans.

PufE is an ortholog of S. cerevisiae Puf6. It contains three PUF repeat motifs and a CPL domain at the C-terminal region. Here, we found that PufE is required for proper fungal development. The $\Delta p u f E$ mutants have increased asexual spore production (Fig. 3) and delayed sexual fruiting body formation (Fig. 4). These results imply that PufE acts as a developmental balancer for asexual and sexual development.

Deletion of PufE reduces sterigmatocystin biosynthesis. We further investigated if PufE also has a role in regulating $A$. nidulans secondary metabolism by analyzing the production of the key secondary metabolite, sterigmatocystin, in $\triangle p u f E$ strains. Sterigmatocystin extracts were separated in a TLC plate. $\triangle p u f E$ mutants produced less sterigmatocystin than control and C' pufE strains (Fig. 5A). To further investigate sterigmatocystin biosynthesis, we checked the mRNA levels of several genes in the sterigmatocystin gene cluster. mRNA levels of aflR, stcA, stcE, and stcU were lower in $\triangle p u f E$ than in the control and C' pufE strains (Fig. 5B). These results suggest that PufE is required for normal sterigmatocystin biosynthesis in A. nidulans.

\section{Role of PufE in A nidulans Conidia}

To further understand PufE developmental roles, we examined pufE mRNA levels throughout the A. nidulans lifecycle (Fig. S1). pufE mRNA levels were high in conidia during asexual development, indicating a role in conidia production. To test this hypothesis, we first analyzed the viability of mutant spores. Loss of pufE slightly decreased spore viability (Fig. 6A). We then determined conidia trehalose content, a key factor for spore viability and stress tolerance. The pufE deletion mutant conidia contained less trehalose than the controls (Fig. 6B). In addition, the pufE deletion mutant conidia were more sensitive to heat stress than those from control and C' pufE strains (Fig. $6 \mathrm{C})$. These results suggest that PufE plays an important role in conidial viability and stress tolerance.

\section{Conclusion}

In this study, we investigated the developmental roles of five A. nidulans PUF proteins. Two of the tested proteins, PufA and PufE, are necessary for proper asexual and sexual development. In addition, PufE plays a vital role in spore viability, thermal stress response, and sterigmatocystin production. These results indicate that PufA and PufE have important roles in development, similar to other eukaryotic PUF proteins. The function of $A$. nidulans PUF proteins in secondary metabolism and stress responses has been studied, but the puf genes do not have a significant effect for stress response and sterigmatocystin production (Figs. S2 and S3). Although the phenotypic analyses were conducted for the puf deletion mutants, the RNA-binding motifs and the targets of $A$. nidulans PUF proteins remain largely unknown. Therefore, further studies are needed to elucidate their biological roles and molecular mechanisms in A. nidulans development and metabolism.

\section{Acknowledgments}

This work was supported by a grant awarded to HSP by the National Research Foundation of Korea (NRF) and funded by the Korean government (NRF-2020R1C1C1004473).

\section{Conflict of Interest}

The authors have no financial conflicts of interest to declare. 


\section{References}

1. McCluskey K, Baker SE. 2017. Diverse data supports the transition of filamentous fungal model organisms into the post-genomics era. Mycology 8: 67-83

2. Etxebeste O, Espeso EA. 2019. Aspergillus nidulans in the post-genomic era: a top-model filamentous fungus for the study of signaling and homeostasis mechanisms. Int. Microbiol. 23: 5-22.

3. Casselton L, Zolan M. 2002. The art and design of genetic screens: filamentous fungi. Nat. Rev. Genet. 3: 683-697.

4. Yu JH. 2010. Regulation of Development in Aspergillus nidulans and Aspergillus fumigatus. Mycobiology 38: 229-237.

5. Park H-S, Yu J-H. 2012. Genetic control of asexual sporulation in filamentous fungi. Curr. Opin. Microbiol. 15: 669-677.

6. Adams TH, Wieser JK, Yu J-H. 1998. Asexual sporulation in Aspergillus nidulans. Microbiol. Mol. Biol. Rev. 62: 35-54.

7. Dyer PS, O'Gorman CM. 2012. Sexual development and cryptic sexuality in fungi: insights from Aspergillus species. FEMS Microbiol. Rev. 36: 165-192.

8. Krijgsheld P, Bleichrodt R, van Veluw GJ, Wang F, Muller WH, Dijksterhuis J, et al. 2013. Development in Aspergillus. Stud. Mycol. 74: $1-29$.

9. Ruger-Herreros C, Rodriguez-Romero J, Fernandez-Barranco R, Olmedo M, Fischer R, Corrochano LM, et al. 2011. Regulation of conidiation by light in Aspergillus nidulans. Genetics 188: 809-822.

10. de Vries RP, Riley R, Wiebenga A, Aguilar-Osorio G, Amillis S, Uchima CA, et al. 2017. Comparative genomics reveals high biological diversity and specific adaptations in the industrially and medically important fungal genus Aspergillus. Genome Biol. 18: 28.

11. Ojeda-Lopez M, Chen W, Eagle CE, Gutierrez G, Jia WL, Swilaiman SS, et al. 2018. Evolution of asexual and sexual reproduction in the aspergilli. Stud. Mycol. 91:37-59.

12. Park H-S, Lee M-K, Han K-H, Kim M-J, Yu J-H. 2019. Developmental Decisions in Aspergillus nidulans, pp. 63-80. In Hoffmeister D, Gressler M (eds.), Biology of the Fungal Cell, 3rd Ed.

13. Adams TH, Boylan MT, Timberlake WE. 1988. brlA is necessary and sufficient to direct conidiophore development in Aspergillus nidulans. Cell 54: 353-362.

14. Park H-S, Ni M, Jeong KC, Kim YH, Yu J-H. 2012. The role, interaction and regulation of the velvet regulator VelB in Aspergillus nidulans. PLoS One 7: e45935.

15. Wu MY, Mead ME, Lee MK, Ostrem Loss EM, Kim SC, Rokas A, et al. 2018. Systematic dissection of the evolutionarily conserved WetA developmental regulator across a genus of filamentous fungi. mBio. 9: $\mathrm{e} 01130$.

16. Wu MY, Mead ME, Lee MK, Neuhaus GF, Adpressa DA, Martien JI, et al. 2021. Transcriptomic, Protein-DNA interaction, and metabolomic studies of VosA, VelB, and WetA in Aspergillus nidulans asexual spores. mBio 12: e03128-20.

17. Park HS, Yu JH. 2017. Velvet regulators in Aspergillus spp. Microbiol. Biotechnol. Lett. 44: 409-419.

18. Drott MT, Bastos RW, Rokas A, Ries LNA, Gabaldon T, Goldman GH, et al. 2020. Diversity of secondary metabolism in Aspergillus nidulans clinical isolates. mSphere 5: e00156-20.

19. Perrone G, Gallo A. 2017. Aspergillus Species and their associated mycotoxins. Methods Mol. Biol. 1542: 33-49.

20. Pfliegler WP, Pocsi I, Gyori Z, Pusztahelyi T. 2019. The Aspergilli and their mycotoxins: Metabolic interactions with plants and the soil biota. Front. Microbiol. 10: 2921.

21. Diaz Nieto CH, Granero AM, Zon MA, Fernandez H. 2018. Sterigmatocystin: A mycotoxin to be seriously considered. Food Chem. Toxicol. 118: 460-470.

22. Brown DW, Yu JH, Kelkar HS, Fernandes M, Nesbitt TC, Keller NP, et al. 1996. Twenty-five coregulated transcripts define a sterigmatocystin gene cluster in Aspergillus nidulans. Proc. Natl. Acad. Sci. USA 93: 1418-1422.

23. Yu JH, Butchko RA, Fernandes M, Keller NP, Leonard TJ, Adams TH. 1996. Conservation of structure and function of the aflatoxin regulatory gene aflR from Aspergillus nidulans and A. flavus. Curr. Genet. 29: 549-555.

24. Fernandes M, Keller NP, Adams TH. 1998. Sequence-specific binding by Aspergillus nidulans AflR, a C6 zinc cluster protein regulating mycotoxin biosynthesis. Mol. Microbiol. 28: 1355-1365.

25. Bayram O, Krappmann S, Ni M, BokJW, Helmstaedt K, Valerius O, et al. 2008. VelB/VeA/LaeA complex coordinates light signal with fungal development and secondary metabolism. Science 320: 1504-1506.

26. Ramamoorthy V, Dhingra S, Kincaid A, Shantappa S, Feng X, Calvo AM. 2013. The putative $\mathrm{C}_{2} \mathrm{H}_{2}$ transcription factor MtfA is a novel regulator of secondary metabolism and morphogenesis in Aspergillus nidulans. PLoS One 8: e74122.

27. Oakley CE, Ahuja M, Sun WW, Entwistle R, Akashi T, Yaegashi J, et al. 2017. Discovery of McrA, a master regulator of Aspergillus secondary metabolism. Mol. Microbiol. 103: 347-365.

28. Lee MK, Son YE, Park HS, Alshannaq A, Han KH, Yu JH. 2020. Velvet activated McrA plays a key role in cellular and metabolic development in Aspergillus nidulans. Sci. Rep. 10: 15075

29. Lunde BM, Moore C, Varani G. 2007. RNA-binding proteins: modular design for efficient function. Nat. Rev. Mol. Cell Biol. 8: 479490.

30. Oliveira C, Faoro H, Alves LR, Goldenberg S. 2017. RNA-binding proteins and their role in the regulation of gene expression in Trypanosoma cruzi and Saccharomyces cerevisiae. Genet. Mol. Biol. 40: 22-30.

31. Hentze MW, Castello A, Schwarzl T, Preiss T. 2018. A brave new world of RNA-binding proteins. Nat. Rev. Mol. Cell Biol. 19:327-341.

32. Kishore S, Luber S, Zavolan M. 2010. Deciphering the role of RNA-binding proteins in the post-transcriptional control of gene expression. Brief Funct. Genomics 9: 391-404

33. Vollmeister E, Feldbrugge M. 2010. Posttranscriptional control of growth and development in Ustilago maydis. Curr. Opin. Microbiol. 13: 693-699.

34. Quenault T, Lithgow T, Traven A. 2011. PUF proteins: repression, activation and mRNA localization. Trends Cell Biol. 21: 104-112.

35. Wang M, Oge L, Perez-Garcia MD, Hamama L, Sakr S. 2018. The PUF Protein Family: Overview on PUF RNA Targets, Biological Functions, and Post Transcriptional Regulation. Int. J. Mol. Sci. 19: 410.

36. Wilinski D, Buter N, Klocko AD, Lapointe CP, Selker EU, Gasch AP, et al. 2017. Recurrent rewiring and emergence of RNA regulatory networks. Proc. Natl. Acad. Sci. USA 114: E2816-E2825.

37. Wilinski D, Qiu C, Lapointe CP, Nevil M, Campbell ZT, Tanaka Hall TM, et al. 2015. RNA regulatory networks diversified through curvature of the PUF protein scaffold. Nat. Commun. 6: 8213.

38. Porter DF, Koh YY, VanVeller B, Raines RT, Wickens M. 2015. Target selection by natural and redesigned PUF proteins. Proc. Natl. Acad. Sci. USA 112: 15868-15873.

39. Gerber AP, Herschlag D, Brown PO. 2004. Extensive association of functionally and cytotopically related mRNAs with Puf family RNA-binding proteins in yeast. PLoS Biol. 2: E79.

40. Haramati O, Brodov A, Yelin I, Atir-Lande A, Samra N, Arava Y. 2017. Identification and characterization of roles for Puf1 and Puf2 proteins in the yeast response to high calcium. Sci. Rep. 7: 3037

41. Gu W, Deng Y, Zenklusen D, Singer RH. 2004. A new yeast PUF family protein, Puf6p, represses ASH1 mRNA translation and is required for its localization. Genes Dev. 18: 1452-1465. 
42. Marhoul JF, Adams TH. 1996. Aspergillus fabM encodes an essential product that is related to poly(A)-binding proteins and activates development when overexpressed. Genetics 144: 1463-1470.

43. Shaw BD, Upadhyay S. 2005. Aspergillus nidulans swoK encodes an RNA binding protein that is important for cell polarity. Fungal Genet. Biol. 42: 862-872.

44. Olszewska A, Krol K, Weglenski P, Dzikowska A. 2007. Arginine catabolism in Aspergillus nidulans is regulated by the rrmA gene coding for the RNA-binding protein. Fungal Genet. Biol. 44: 1285-1297.

45. Krol K, Morozov IY, Jones MG, Wyszomirski T, Weglenski P, Dzikowska A, et al. 2013. RrmA regulates the stability of specific transcripts in response to both nitrogen source and oxidative stress. Mol. Microbiol. 89: 975-988.

46. Soukup AA, Fischer GJ, Luo J, Keller NP. 2017. The Aspergillus nidulans Pbp1 homolog is required for normal sexual development and secondary metabolism. Fungal Genet. Biol. 100: 13-21.

47. Park HS, Yu JH. 2012. Multi-copy genetic screen in Aspergillus nidulans. Methods Mol. Biol. 944: 183-190.

48. Park HS, Nam TY, Han KH, Kim SC, Yu JH. 2014. VelC positively controls sexual development in Aspergillus nidulans. PLoS One 9: e89883.

49. Yu JH, Hamari Z, Han KH, Seo JA, Reyes-Dominguez Y, Scazzocchio C. 2004. Double-joint PCR: a PCR-based molecular tool for gene manipulations in filamentous fungi. Fungal Genet. Biol. 41: 973-981.

50. Kim MJ, Jung WH, Son YE, Yu JH, Lee MK, Park HS. 2019. The velvet repressed vidA gene plays a key role in governing development in Aspergillus nidulans. J. Microbiol. 57: 893-899.

51. Park HS, Lee MK, Kim SC, Yu JH. 2017. The role of VosA/VelB-activated developmental gene vadA in Aspergillus nidulans. PLoS One 12: 0177099

52. Ni M, Yu J-H. 2007. A novel regulator couples sporogenesis and trehalose biogenesis in Aspergillus nidulans. PLoS One 2: e970.

53. Sarikaya Bayram O, Bayram O, Valerius O, Park HS, Irniger S, Gerke J, et al. 2010. LaeA control of velvet family regulatory proteins for light-dependent development and fungal cell-type specificity. PLoS Genet. 6: e1001226.

54. Galagan JE, Calvo SE, Cuomo C, Ma LJ, Wortman JR, Batzoglou S, et al. 2005. Sequencing of Aspergillus nidulans and comparative analysis with $A$. fumigatus and A. oryzae. Nature 438: 1105-1115.

55. Shaaban MI, Bok JW, Lauer C, Keller NP. 2010. Suppressor mutagenesis identifies a velvet complex remediator of Aspergillus nidulans secondary metabolism. Eukaryot. Cell 9: 1816-1824.

56. Kwon NJ, Shin KS, Yu JH. 2010. Characterization of the developmental regulator FlbE in Aspergillus fumigatus and Aspergillus nidulans. Fungal Genet. Biol. 47: 981-993. 